\title{
Collapse and control of the $\mathrm{MnAu}_{2}$ spin-spiral state through pressure and doping
}

\author{
J. K. Glasbrenner \\ National Research Council/Code 6393, Naval Research Laboratory, Washington, DC 20375, USA
}

\begin{abstract}
$\mathrm{MnAu}_{2}$ is a spin-spiral material with in-plane ferromagnetic Mn layers that form a screw-type pattern around a tetragonal $c$ axis. The spiral angle $\theta$ was shown using neutron diffraction experiments to decrease with pressure, and in later studies it was found to suffer a collapse to a ferromagnetic state above a critical pressure, although the two separate experiments did not agree on whether this phase transition is first or second order. To resolve this contradiction, we use density functional theory calculations to investigate the spiral state as a function of pressure, charge doping, and also electronic correlations via a Hubbard-like $U$. We fit the results to the one-dimensional $J_{1}-J_{2}-J_{3}-J_{4}$ Heisenberg model, which predicts either a first- or second-order spiral-to-ferromagnetic phase transition for different regions of parameter space. At ambient pressure, $\mathrm{MnAu}_{2}$ sits close in parameter space to a dividing line separating first- and second-order transitions, and a combination of pressure and electron doping shifts the system from the first-order region into the second-order region. Our findings demonstrate that the contradiction in pressure experiments regarding the kind of phase transition are likely due to variations in sample quality. Our results also suggest that $\mathrm{MnAu}_{2}$ is amenable to engineering via chemical doping and to controlling $\theta$ using pressure and gate voltages, which holds potential for integration in spintronic devices.
\end{abstract}

\section{INTRODUCTION}

The tetragonal material $\mathrm{MnAu}_{2}$ is one of the oldest known spin-spiral materials [1-3, with a Néel temperature of $T_{N}=363 \mathrm{~K} \mathrm{[1]} \mathrm{and} \mathrm{a} \mathrm{local} \mathrm{Mn}$ moment of $3.5 \mu_{B}$ [3, 4]. The magnetic ground state consists of inplane ferromagnetic Mn layers with a screw-type pattern around the tetragonal $c$ axis, which can be described using the spiral angle $\theta$ [this is equivalent to the wave vector $\mathbf{q}=\left(0,0, q_{z}\right)$, where $\left.\theta=q_{z} c / 2\right]$. Neutron diffraction measurements [3, 5, 6] find that $\theta$ has a weak dependence on temperature, increasing slightly with increasing temperature $\left[\theta(77 \mathrm{~K})=47^{\circ}\right.$ and $\left.\theta(295 \mathrm{~K})=51^{\circ}\right]$. The spiral state is known to collapse to a metamagnetic fan-like configuration when placed in a $\sim 10 \mathrm{kOe}$ magnetic field at room temperature [1, 2], which led to a revival of interest in $\mathrm{MnAu}_{2}$ when it was found that this gives rise to a giant magnetoresistive effect [4].

There are several microscopic mechanisms that can induce spin spirals. In itinerant systems, both Fermi surface nesting [7] and the Ruderman-Kittel-Kasuya-Yosida (RKKY) interaction [8] 10] can lead to spiral formation. The Dzyaloshinsky-Moriya (DM) interaction [11, 12], which occurs in materials without an inversion center $\left(\mathrm{MnAu}_{2}\right.$ has an inversion center, which rules it out as a possible mechanism), can also induce spirals and moment canting, but due to the interaction's relativistic origin it is more important in materials with heavy elements. It can still be competitive in lighter transition metals, such as $\mathrm{MnSi}$ [13 15], but the spirals will have a long wavelength due to weak relativistic effects. Finally, frustration, which can be geometric or magnetic, can encourage noncollinearity and lead to the formation of spirals.

The spin spirals in $\mathrm{MnAu}_{2}$ are understood to be a consequence of magnetic frustration, which is commonly modeled using a one-dimensional Heisenberg model with first and second neighbor interplanar couplings (the $J_{1}-$ $J_{2}$ model) [16, 17]. In this model, the spiral state is stable when $J_{1} \neq 0, J_{2}>0$, and $\left|J_{1}\right|<4\left|J_{2}\right|[16]$. The validity of applying this model to $\mathrm{MnAu}_{2}$ was confirmed using density functional theory (DFT) calculations [18, 19]. The origin of the magnetic frustration was traced to a competition between two exchange mechanisms, nearestneighbor superexchange and a transferred RKKY-like interaction, and electronic correlations were found to play an essential role in suppressing the RKKY-like interaction, which is necessary to satisfy the $\left|J_{1}\right|<4\left|J_{2}\right|$ inequality [19].

The spiral angle $\theta$ is sensitive to pressure, with neutron diffraction measurements determining that $\theta(P)$ decreases with applied pressure $\left[\theta(0)=50.7^{\circ}\left(47.0^{\circ}\right)\right.$ decreasing to $\theta(8.83 \mathrm{kbar})=41.8^{\circ}\left(40.5^{\circ}\right)$ at a temperature of $295 \mathrm{~K}(77 \mathrm{~K})$ ] [5]. Measurements of the critical magnetic field $H_{c}$ for the spiral-to-ferromagnetic transition as a function of pressure, when extrapolated to zero external field, implied that the spiral state should collapse to ferromagnetism at $\sim 12 \mathrm{kbar}[20$. The pressureinduced spiral-to-ferromagnetic transition was confirmed by subsequent experiments using inductance measurements [21], electrical resistivity measurements, and measurements of $H_{c}$ [22], although the two reports disagreed on the order of the phase transition. In Ref. 21, the transition is of the second kind and occurs over a pressure range of 12-20 kbar, while in Ref. 22 it is of the first kind and occurs at $13 \mathrm{kbar}$. This contradiction was never resolved and remained an open question.

The $J_{1}-J_{2}$ Heisenberg model, which can explain the stability of the spiral state, predicts that the transition must be of the second kind. However, general investigations of the classical one-dimensional Heisenberg model show that including the third nearest-neighbor term $J_{3}$ leads to regions of parameter space with first order phase transitions [23, 24]. Further neighbors, such as $J_{4}$, change the area of parameter space where first-order transitions occur [24]. It follows then that if a system were to sit near the boundary in parameter space separating first- and 
second-order phase transitions, then variations in material quality could shift the system towards one region or the other.

We argue that this is the case for $\mathrm{MnAu}_{2}$. We support this claim using DFT calculations to simulate the effects of pressure, charge doping, and variation in the strength of electronic correlations via a Hubbard-like $U$. We calculate the magnetic energies and fit them with a classical, one-dimensional Heisenberg model with coupling between the first four nearest-neighbor Mn planes. We confirm that ideal, stoichiometric $\mathrm{MnAu}_{2}$ sits close to a boundary separating first- and second-order phase transitions, and that varying the pressure and doping shifts $\mathrm{MnAu}_{2}$ across this critical boundary. This suggests that the two pressure experiments are not in conflict, but instead the $\mathrm{MnAu}_{2}$ samples from each are located in two separate portions of phase space. The spiral angle $\theta$ is also found to be responsive to applied pressure and doping, which holds promise for integration of $\mathrm{MnAu}_{2}$ into spintronic devices.

\section{COMPUTATIONAL DETAILS}

We employed DFT to solve the electronic structure of $\mathrm{MnAu}_{2}$ and calculate the total energy of magnetic configurations. To perform these calculations, we used the all-electron code ELK [25, which is an implementation of the full-potential linear augmented planewave method, and also projector augmented wave potentials implemented in the code VASP [26, 27]. We used the local spin-density approximation (LSDA) 28 for our calculations, and for correlation effects we used the DFT $+U$ method in the fully localized limit [29], in which we introduce a Hubbard-like $U$ on the $3 d$ orbitals of the $\mathrm{Mn}$ atoms. We used two values of $U, U=3.12 \mathrm{eV}$ and $4.7 \mathrm{eV}$, and $J$ was set to $0.7 \mathrm{eV}$.

$\mathrm{MnAu}_{2}$ has a tetragonal crystal structure with $I 4 / \mathrm{mmm}$ space group symmetry. The experimental lattice parameters are $a=3.370 \AA$ and $c / a=2.599$, yielding a volume of $49.741 \AA^{3}$ /formula unit, and the Wyckoff positions for $\mathrm{Mn}$ and $\mathrm{Au}$ are $2 a$ and $4 e$, respectively, with the internal experimental height for $\mathrm{Au} z_{A u}=0.34$ (fractional coordinates) [30, 31. To simulate pressure, we began with the $\mathrm{MnAu}_{2}$ unit cell from experiment with tetragonal symmetry and varied the lattice parameters $a$ and $c$ to set the volume [32]. We then fixed the volume and performed structural relaxations in VASP to optimize the $c / a$ ratio and the internal parameter $z_{A u}$. After the relaxation, we performed total energy calculations in VASP with the tetrahedron method and calculated the pressure using the formula $P=-d E / d V$. We then imported the relaxed structures into ELK for calculating the magnetic energies.

We employed two methods to simulate charge doping, (1) adding electrons directly to the system along with a uniform positive charge background (hereafter referred to as "charge dosing"), and (2) using the virtual crystal approximation (VCA). The VCA involves replacing the $\mathrm{Au}$ ions with fictitious ions of fractional charge in order to add electrons or holes to the system. We used the experimental lattice parameters of $\mathrm{MnAu}_{2}$ in our doping calculations, while for calculations with simultaneous charge doping and applied pressure we used the relaxed cells obtained with VASP.

We used the spin spiral method implemented in ELK to calculate the total energy as a function of the spiral angle. This method defines a wave vector $\mathbf{q}=\left(0,0, q_{z}\right)$ that is applied to a primitive cell to simulate spirals. In our previous study of $\mathrm{MnAu}_{2}$ [19, we found good agreement between the calculated energies obtained via this method and noncollinear calculations of spirals in supercells. In this study, our procedure was to calculate the energy as a function of the wave vector for the range $0<q_{z}<2 \pi / c$ as we varied the pressure, charge doping, and Hubbard $U$. We then used $\theta=q_{z} c / 2$ to convert the wave vector to an angle.

Convergence for the spin spiral calculations was carefully checked in the primitive cells, and the following parameters were necessary to achieve convergence: k-mesh $=16 \times 16 \times 16$, nempty $=8, \operatorname{rgkmax}=8.0$, gmaxvr $=$ 12 , lmaxapw $=8$, and the smearing function width was set to $0.0001 \mathrm{Ha}$.

\section{SUMMARY OF 1D HEISENBERG MODEL}

The magnetic interactions in $\mathrm{MnAu}_{2}$ are typically modeled using the Heisenberg model:

$$
H=\sum_{i \neq j} J_{i j} \hat{\mathbf{m}}_{i} \cdot \hat{\mathbf{m}}_{j}
$$

Note that $J_{i j}=\bar{J}_{i j}|\mathbf{m}|^{2}$, where $\bar{J}_{i j}$ is the magnetic coupling and $|\mathbf{m}|$ is the moment amplitude. DFT calculations confirm that $\mathrm{Mn}$ is in the high spin state with $S=5 / 2$, which makes the classical Heisenberg model suitable for studying the magnetic interaction. The magnetic moment is also assumed to be constant, which is reasonable as the Mn moment amplitudes do not vary by much as a function of $\theta$.

The spiral state of $\mathrm{MnAu}_{2}$ is completely described by the parameter $\theta$. As a result, we can simplify the Heisenberg model to one dimension:

$$
H=\text { const. }+\sum_{n=1}^{N} J_{n} \cos (n \theta)
$$

The interplanar coupling determines the spiral state stability, and below we discuss the phase diagram for the models which include two $\left(J_{1}-J_{2}\right.$ model $)$, three $\left(J_{1}-J_{2}-J_{3}\right.$ model $)$, and four $\left(J_{1}-J_{2}-J_{3}-J_{4}\right.$ model $)$ nearest-neighbors in Eq. (2).

The $J_{1}-J_{2}$ model is the simplest possible model with a stable spiral state, which occurs when $J_{1} \neq 0, J_{2}>0$, and $\left|J_{1}\right|<4\left|J_{2}\right|$. The angle $\theta$ is plotted as a function 

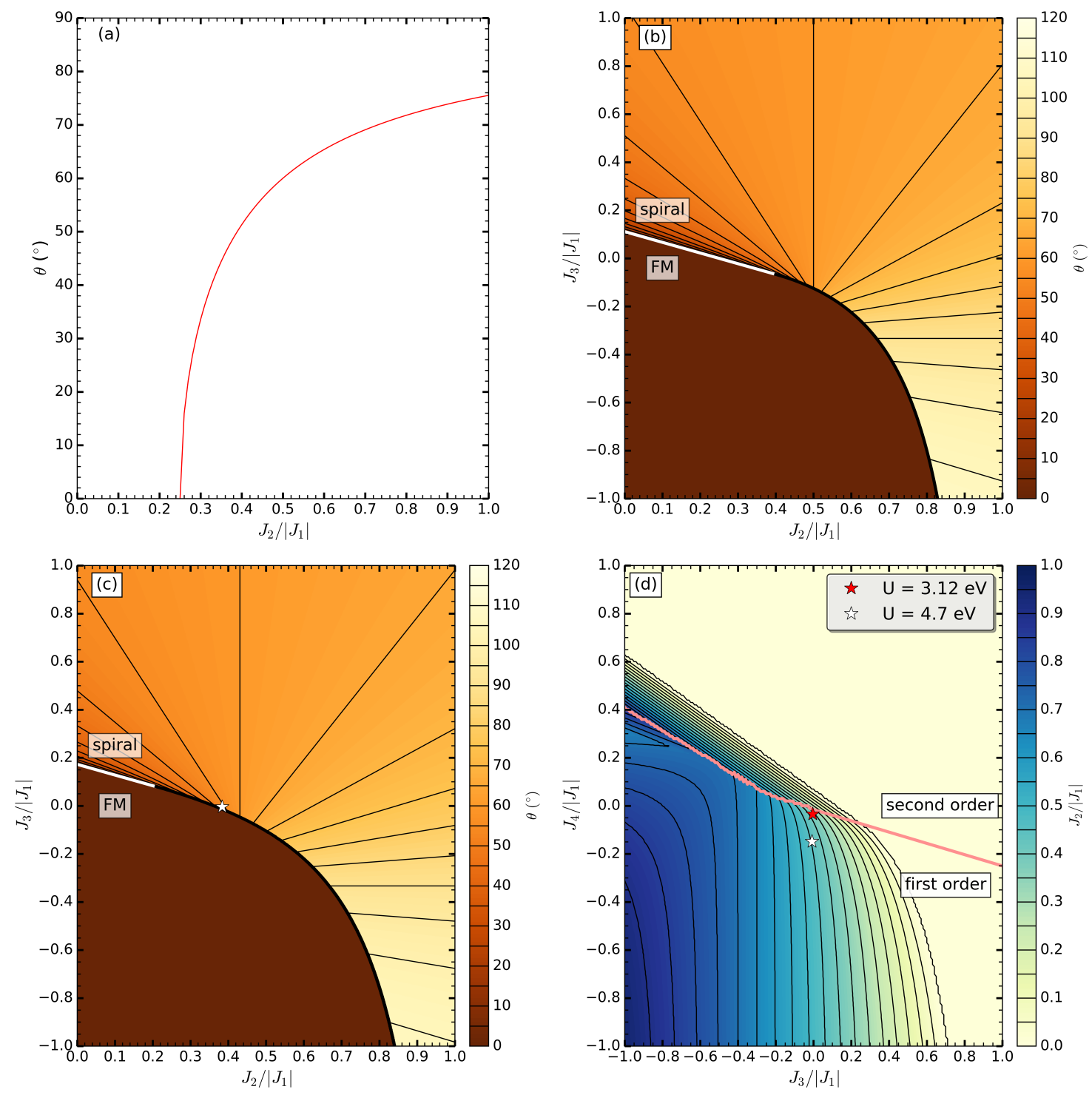

FIG. 1. (Color online) Phase diagrams for the one-dimensional Heisenberg model of Eq. (1). (a) The phase diagram of the $J_{1}-J_{2}$ model. (b) The phase diagram of the $J_{1}-J_{2}-J_{3}$ model, where the contour plot corresponds to $\theta$. The thick black line indicates a first-order phase transition and the thick white line indicates a second-order transition. (c) The phase diagram of the $J_{1}-J_{2}-J_{3}-J_{4}$ model for $J_{4} /\left|J_{1}\right|=-0.0346$, plotted in the same manner as panel (b). The white star indicates where ideal stoichiometric $\mathrm{MnAu}_{2}$ with $U=3.12 \mathrm{eV}$ sits in the phase diagram. (d) The critical values of $J_{2} /\left|J_{1}\right|$ (contour plot), $J_{3} /\left|J_{1}\right|$, and $J_{4} /\left|J_{1}\right|$ for the spiral-to-ferromagnetic phase transition. The red line divides parameter space into two regions, where first-order phase transitions occur north of the line, and second-order transitions occur south of it. The red and white stars represent where ideal, stoichiometric $\mathrm{MnAu}_{2}$ sits for $U=3.12 \mathrm{eV}$ and $U=4.7 \mathrm{eV}$, respectively.

of $J_{2} /\left|J_{1}\right|$ in Fig. 1(a), and the spiral-to-ferromagnetic phase transition is of second order. What has not been appreciated in previous studies of $\mathrm{MnAu}_{2}$ is that this model cannot explain how the turn angle could collapse under pressure in a first-order transition, as measured in Ref. 22,

Including the third nearest-neighbor coupling yields the $J_{1}-J_{2}-J_{3}$ model, which can be solved analytically (see Supplemental Material [33]) 23, 24. The phase diagram of $\theta$ for this model as a function of $J_{2} /\left|J_{1}\right|$ and
$J_{3} /\left|J_{1}\right|$ is depicted in Fig. 1 $1(\mathrm{~b})$. There are two regions in the parameter space, the ferromagnetic region in the lower left and the spiral region in the rest of the diagram, with the contour plot corresponding to the magnitude of $\theta$. The phase boundaries separating the two regions are [23]:

$$
J_{3}=\left\{\begin{array}{ll}
\frac{1}{9}\left(\left|J_{1}\right|-4 J_{2}\right) & 0 \leq J_{2} /\left|J_{1}\right| \leq 2 / 5 \\
\frac{J_{2}^{2}}{4\left(J_{2}-\left|J_{1}\right|\right)} & J_{2} /\left|J_{1}\right|>2 / 5
\end{array} .\right.
$$

Importantly, this model contains both first- and second- 


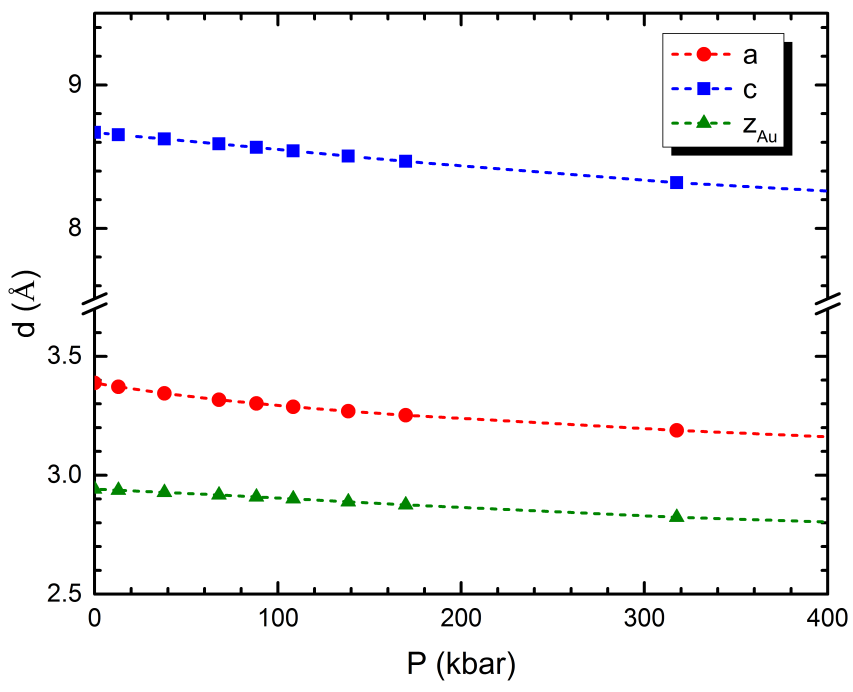

FIG. 2. (Color online) $\mathrm{MnAu}_{2}$ lattice parameters as a function of pressure.

order phase transitions, which are distinguished by the kind of degeneracy that occurs on the borders in Eq. (3). A first-order phase transition occurs when there is a degeneracy between the ferromagnetic solution $(\theta=0)$ and a finite angle $(\theta>0)$, while the second order phase transition corresponds to a smooth, continuous connection between ferromagnetic and spin-spiral regions, and is found by taking a second-order Maclaurin series expansion of Eq. (2) and equating it with the ferromagnetic solution in the limit $\theta \rightarrow 0$. Second-order transitions correspond with the $0 \leq J_{2} /\left|J_{1}\right| \leq 2 / 5$ result and are depicted as the thick white line in Fig. 1(b), while first order transitions correspond to the $J_{2} /\left|J_{1}\right|>2 / 5$ result and are depicted as the thick black line. From this, it is clear that a small ferromagnetic coupling between third-neighbor planes (relative to $\left|J_{1}\right|$ ) can change the order of the phase transition.

The analysis is more complicated for four or more neighbor couplings since an analytic solution for $\theta$ is not available. Despite this, some properties can still be analytically solved (see Supplemental Material [33]) [24]. The phase boundary for second order transitions is $J_{2}=\left(\left|J_{1}\right|-9 J_{3}-16 J_{4}\right) / 4$. The criterion for a first order phase transition is

$$
J_{3} /\left|J_{1}\right|<-\frac{1+64 J_{4} /\left|J_{1}\right|}{15} .
$$

Note that the expression for the second order phase boundary and Eq. (4) are valid when $J_{3}, J_{4}>0$. If one of the parameters is negative, then the expressions still hold as long as the antiferromagnetic couplings dominate [24], such as $J_{3}>0, J_{4}<0$, and $\left|J_{3}\right| \gg\left|J_{4}\right|$. What's clear is that a relatively small fourth neighbor coupling can have a dramatic effect on the location of the first order and second order phase boundaries. To illustrate this, we numerically calculate the phase diagram for the $J_{1}-J_{2}-J_{3}-J_{4}$ model with $J_{4} /\left|J_{1}\right|=-0.0346$, which we plot in Fig. 1 (c). Comparing panels (b) and (c), we clearly see that the length of the second order phase boundary (white line) has changed, despite the relative weakness of the fourth neighbor coupling. We also calculate the exchange parameters for ideal, stoichiometric $\mathrm{MnAu}_{2}$ with $U=3.12 \mathrm{eV}$, which are $J_{2} /\left|J_{1}\right|=0.3840$, $J_{3} /\left|J_{1}\right|=-0.0043, J_{4} /\left|J_{1}\right|=-0.0346$, and indicate where $\mathrm{MnAu}_{2}$ sits with the white circle. The material sits very close to the first order transition, and a small variation in $J_{3} /\left|J_{1}\right|$ would drive the system towards ferromagnetism.

In Fig. 1(d) we plot a contour map of the critical values of $J_{2} /\left|J_{1}\right|, J_{3} /\left|J_{1}\right|$, and $J_{4} /\left|J_{1}\right|$ for the spiral-toferromagnetic transition in the one-dimensional $J_{1}-J_{2}-$ $J_{3}-J_{4}$ model. We only consider $J_{2} \geq 0$ in this figure. The contour plot shows the surface in $\left(J_{2} /\left|J_{1}\right|, J_{3} /\left|J_{1}\right|\right.$, $\left.J_{4} /\left|J_{1}\right|\right)$ space that separates ferromagnetic and spiral order. For example, if you calculate the exchange parameters for $\mathrm{MnAu}_{2}$ and those parameters place the system "inside" the surface $\left(J_{2}<J_{2}^{c}\right)$, then the ground state magnetic order is ferromagnetic. If instead the parameters place $\mathrm{MnAu}_{2}$ on or outside the surface $\left(J_{2} \geq J_{2}^{c}\right)$, then the magnetic order will be a spiral state. Note that the light yellow region corresponding to $J_{2}^{c}=0$ means that the system has spiral order for all $J_{2} \geq 0$. Finally, if you can tune the parameters, such as by applying pressure or adding holes/electrons, then the system can cross the surface and undergo a phase transition.

As we saw in panels (b) and (c) of Fig. 1. phase transitions can either be first or second order. The parameters $\left(J_{3} /\left|J_{1}\right|, J_{4} /\left|J_{1}\right|\right)$ control the kind of phase transition that occurs when you cross the surface depicted in Fig. 1(d). The red line separates the first- and secondorder transition regions. If you cross the surface while north of the line, then the phase transition is second order; if you cross the surface while south of the line, then the phase transition is first order. Having established this, it is now instructive to indicate where ideal, stoichiometric $\mathrm{MnAu}_{2}$ sits in $\left(J_{3} /\left|J_{1}\right|, J_{4} /\left|J_{1}\right|\right)$ space for $U=3.12 \mathrm{eV}$ (red star) and $U=4.7 \mathrm{eV}$ (white star). For both systems, $J_{2}>J_{2}^{c}$, so they have spiral order. What is striking here is how close $\mathrm{MnAu}_{2}$ sits to the dividing line, especially when $U=3.12 \mathrm{eV}$. This shows that changes in the exchange parameters induced through pressure, doping, or impurities could place the system north of the red line. Since the system sits close to the dividing line, this could explain the discrepancy in experimental pressure studies discussed in Sec. I.

One final point to note is that this analysis assumed that the local moments always have an in-plane orientation. Neutron diffraction experiments confirm that spin spirals in $\mathrm{MnAu}_{2}$ are oriented in-plane [2], and our calculations of the magnetocrystalline anisotropy energy for $\mathrm{MnAu}_{2}$ with $U=3.12 \mathrm{eV}$ give $E(001)-E(100)=$ $0.35 \mathrm{meV}$ 34, indicating the in-plane direction is the preferred axis. Yet, it is less clear what would happen on the first order transition boundary, where zero and finite angle configurations are degenerate. We checked 

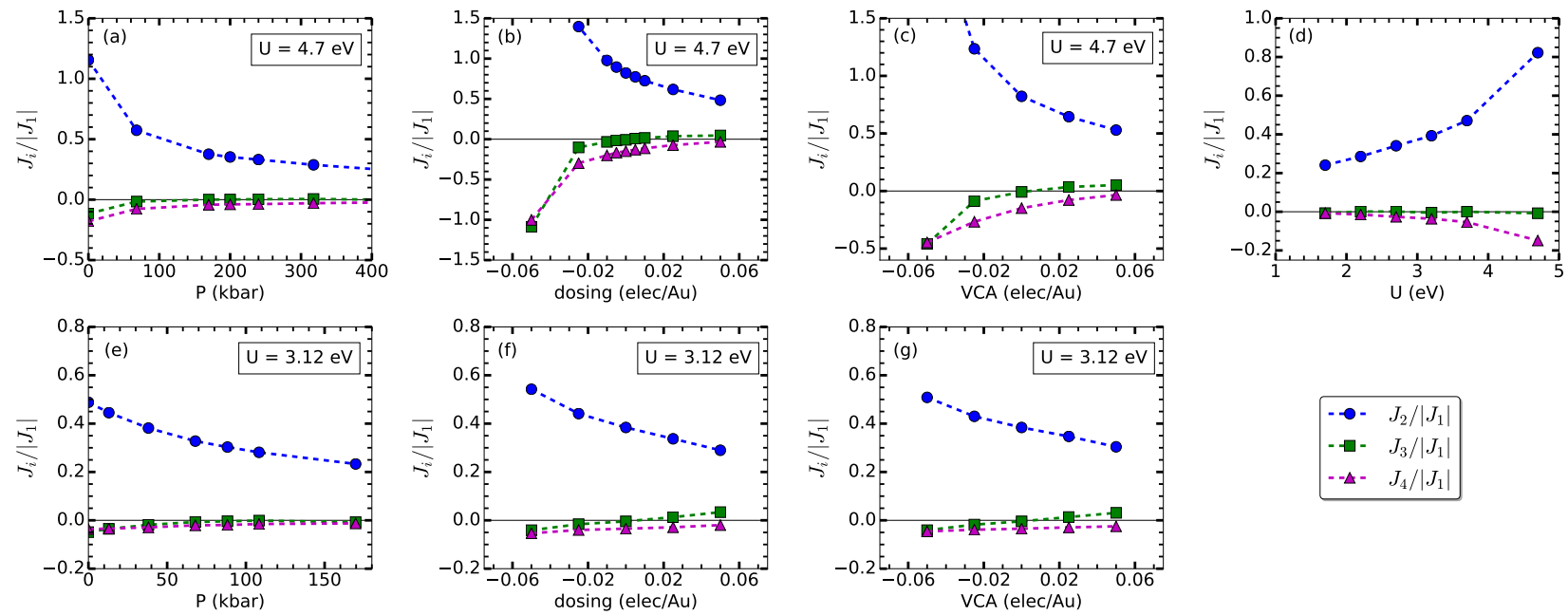

FIG. 3. (Color online) The dependence of the exchange parameters $J_{2} /\left|J_{1}\right|, J_{3} /\left|J_{1}\right|$, and $J_{4} /\left|J_{1}\right|$, see legend, as a function of pressure, doping, and $U$. In the charge dosing and VCA panels, positive values on the horizontal axis correspond to electron doping and negative values to hole doping. (a) Pressure dependence with $U=4.7 \mathrm{eV}$. (b) Charge dosing dependence with $U=4.7 \mathrm{eV}$. (c) VCA dependence with $U=4.7 \mathrm{eV}$. (d) Dependence on Hubbard $U$. (e) Pressure dependence with $U=3.12 \mathrm{eV}$. (f) Charge dosing dependence with $U=3.12 \mathrm{eV}$. (g) VCA dependence with $U=3.12 \mathrm{eV}$.

this possibility by considering conical solutions. We added a canted angle $\varphi$ and a uniaxial magnetocrystalline anisotropy term to Eq. (2),

$$
\begin{aligned}
& E(\theta, \varphi)=\text { const }+D \sin ^{2}(\varphi)+ \\
& \qquad \sum_{n=1}^{m} J_{n}\left[1-2 \sin ^{2}(\varphi) \sin ^{2}\left(\frac{n \theta}{2}\right)\right] .
\end{aligned}
$$

In this expression, the angles $\theta$ and $\varphi$ remain decoupled and the solutions for $\theta$ are unaffected by canting and simple uniaxial magnetocrystalline anisotropy. If we now restrict ourselves to the first-order phase transition boundary, the energy difference between the conical spirals and the ferromagnetic configuration is

$$
E(\theta, \varphi)-E\left(0, \varphi^{\prime}\right)=D\left[\sin ^{2}(\varphi)-\sin ^{2}\left(\varphi^{\prime}\right)\right]
$$

Therefore in the absence of magnetocrystalline anisotropy there is a continuous degeneracy of the canting angle. This degeneracy is lifted with the magnetocrystalline anisotropy term, although we should note that the magnetocrystalline anisotropy parameter is small, $D=0.35 \mathrm{meV} \sim 4 \mathrm{~K}$, and since the pressure experiments [5, 21, 22] were performed at temperatures of $77 \mathrm{~K}$ and at room temperature, it is possible for the ferromagnetic orientation to align along the $c$ axis. It could also be used as a continuous pathway to make $\theta$ drop to zero (at finite $\theta$ the moments can align parallel to $c$ while on the first-order boundary, and then as you move away from the boundary they can drop back to being in-plane with $\theta=0$ ). This means that the phase transition may not be truly first order. However, it should be noted that such a transition entails a rapid change in the canting angle, which would be difficult to distinguish from a first-order transition in experiment. For the rest of the discussion, we will continue to refer to this as a first-order transition with the caveat that it may correspond to a rapid change in the canting angle. Regardless, it remains distinct from the second-order transition, where $\theta$ continuously decreases to zero.

\section{DFT RESULTS AND DISCUSSION}

Having established in Sec. III that $\mathrm{MnAu}_{2}$ sits close to a dividing line that separates first and second order phase transitions, we now use DFT to perform a systematic set of spin-spiral calculations to simulate the effects of pressure and doping, and the effect of varying $U$. This will reveal how applying pressure and doping makes $\mathrm{MnAu}_{2}$ evolve in parameter space. Each set of spin spiral calculations results in a $E(\theta)$ curve, and all curves are similar to what was obtained in our previous report [19]. Each curve was fitted using the $J_{1}-J_{2}-J_{3}-J_{4}$ model, and from these we extract the exchange constants (see Supplemental Material [33]).

It should be noted that these calculations and their subsequent analysis are all done at zero temperature, a choice that we should justify. Measurements of the saturation magnetization at low temperatures $(T \leq 10 \mathrm{~K})$ and at room temperature [35, 36], along with measurements of the susceptibility and the critical magnetic field $H_{c}$ [35], show a very weak dependence on temperature. As discussed in Sec. [1 the temperature dependence of the spiral angle as a function of pressure, obtained using neutron diffraction measurements at $77 \mathrm{~K}$ and at room temperature, is also weak [5, 6]. The weak temperature dependence of these material parameters suggests that 


\begin{tabular}{|c|c|c|c|c|c|c|c|c|c|c|}
\hline $\begin{array}{c}\text { Pressure } \\
\text { (kbar) }\end{array}$ & $J_{1}$ & $\begin{array}{l}J_{2} \\
(\mathrm{meV}\end{array}$ & $V)^{J_{3}}$ & $J_{4}$ & $\begin{array}{c}\text { Doping } \\
(\mathrm{elec} / \mathrm{Au})\end{array}$ & Type & $J_{1}$ & $\begin{array}{l}J_{2} \\
(\mathrm{mel}\end{array}$ & V) & $J_{4}$ \\
\hline \multicolumn{11}{|c|}{$U=3.12 \mathrm{eV}$} \\
\hline 0.000 & -20.1394 & 9.8339 & -0.9823 & -0.7333 & -0.050 & Dosing - & -12.5109 & $6.7832-$ & -0.5134 & -0.6678 \\
\hline 12.977 & -22.3635 & 9.9571 & -0.7869 & -0.7692 & & VCA - & -14.1347 & $7.1857-$ & -0.5786 & -0.6446 \\
\hline 38.109 & -26.5047 & $10.1130-$ & -0.4949 & -0.7711 & -0.025 & Dosing - & -16.2810 & $7.1840-$ & -0.2603 & -0.6489 \\
\hline 67.892 & -31.1437 & $10.2032-$ & -0.2159 & -0.6435 & & $\mathrm{VCA}$ & -17.1181 & $7.3614-$ & -0.3046 & -0.6662 \\
\hline 88.298 & -34.1544 & $10.3499-$ & -0.1301 & -0.6544 & 0.000 & Dosing - & -20.2505 & $7.7762-$ & -0.0861 & -0.7000 \\
\hline 108.353 & -37.0067 & $10.4056-$ & -0.0440 & -0.5633 & & $\mathrm{VCA}$ & -20.2505 & $7.7762-$ & -0.0861 & -0.7000 \\
\hline \multirow[t]{4}{*}{169.709} & -44.9705 & $10.4817-$ & -0.3415 & -0.5701 & 0.025 & Dosing - & -24.5855 & 8.2909 & 0.3200 & -0.7065 \\
\hline & & & & & & VCA - & -23.5647 & 8.1775 & 0.3180 & -0.6913 \\
\hline & & & & & 0.050 & Dosing - & -29.1444 & 8.4463 & 0.9843 & -0.5759 \\
\hline & & & & & & $\mathrm{VCA}$ & -26.9893 & 8.2059 & 0.8370 & -0.6678 \\
\hline \multicolumn{11}{|c|}{$U=4.7 \mathrm{eV}$} \\
\hline 0.000 & -5.5435 & $6.4037-$ & -0.6415 & $\begin{array}{l}-0.9926 \\
\end{array}$ & -0.050 & Dosing & -0.6574 & $3.6749-$ & -0.7146 & -0.6583 \\
\hline 67.892 & -13.4226 & 7.7049 & -0.2128 & -1.0301 & & $\mathrm{VCA}$ & -1.6202 & $4.0940-$ & -0.7442 & -0.7241 \\
\hline 169.709 & -24.2692 & 9.1221 & 0.0078 & -1.0323 & -0.025 & Dosing & -3.1144 & $4.3517-$ & -0.3170 & -0.9235 \\
\hline 200.000 & -27.4309 & 9.6846 & 0.0290 & -1.0575 & & $\mathrm{VCA}$ & -3.6679 & $4.5317-$ & -0.3207 & -0.9799 \\
\hline 240.000 & -31.4087 & 10.4061 & 0.1081 & -1.1649 & -0.010 & Dosing & -4.8464 & $4.7346-$ & -0.1601 & -0.9704 \\
\hline \multirow[t]{9}{*}{317.691} & -39.3228 & 11.3247 & 0.2179 & -1.1539 & -0.005 & Dosing & -5.4763 & $4.9005-$ & -0.0754 & -0.9132 \\
\hline & & & & & 0.000 & Dosing & -6.1229 & $5.0350-$ & -0.0480 & -0.9103 \\
\hline & & & & & & $\mathrm{VCA}$ & -6.1229 & $5.0350-$ & -0.0480 & -0.9103 \\
\hline & & & & & 0.005 & Dosing & -6.7465 & 5.2203 & 0.0486 & -0.8760 \\
\hline & & & & & 0.010 & Dosing & -7.4455 & 5.4008 & 0.1156 & -0.8414 \\
\hline & & & & & 0.025 & Dosing & -9.6007 & 5.9304 & 0.3580 & -0.7010 \\
\hline & & & & & & $\mathrm{VCA}$ & -8.8932 & 5.7378 & 0.3137 & -0.6957 \\
\hline & & & & & 0.050 & Dosing - & -13.4470 & 6.5046 & 0.6154 & -0.4418 \\
\hline & & & & & & $\mathrm{VCA}$ & -11.7329 & 6.2087 & 0.6055 & -0.4044 \\
\hline $\begin{array}{c}U \\
(\mathrm{eV})\end{array}$ & $J_{1}$ & $\begin{array}{l}J_{2} \\
(\mathrm{meV}\end{array}$ & V) ${ }^{J_{3}}$ & $J_{4}$ & & & & & & \\
\hline 1.7 & -37.7314 & $9.0945-$ & -0.2716 & -0.2806 & & & & & & \\
\hline 2.2 & -31.4231 & 8.9775 & 0.0325 & -0.4564 & & & & & & \\
\hline 2.7 & -25.0395 & 8.5043 & 0.0267 & -0.6542 & & & & & & \\
\hline 3.2 & -19.4161 & 7.6273 & -0.0997 & -0.7022 & & & & & & \\
\hline 3.7 & -14.3696 & 6.7687 & -0.0028 & -0.7722 & & & & & & \\
\hline 4.7 & -6.1229 & 5.0350 & -0.0480 & -0.9103 & & & & & & \\
\hline
\end{tabular}

TABLE I. Values of the exchange parameters as a function of pressure, charge dosing, the VCA, and $U$. Pressure and doping results for $U=3.12 \mathrm{eV}$ and $U=4.7 \mathrm{eV}$ are reported in the first two sections of the table, where the first five columns are the pressure results and the last six columns are the doping results. The "type" column indicates whether the doping was performed using charge dosing or the VCA. The parameters as a function of $U$ are reported in the last section of the table.

the internal energy determines the important qualitative features of the magnetic phase diagram of $\mathrm{MnAu}_{2}$, and that including thermal fluctuations will result in only minor changes to the area of the spiral and ferromagnetic regions and the length of the first and second order phase boundaries. Therefore, it is reasonable to expect that our conclusions about the spiral-to-ferromagnetic phase transition obtained at zero temperature hold at finite temperatures.

For the pressure calculations, we constrained the volume of our cell and relaxed the structure as described in Sec. [II] and then calculated the pressure using the formula $P=-d E / d V$ (see Supplemental Material [33]). Using the LSDA functional during relaxation results in overbinding, and so here the equilibrium volume is predicted to be $6 \%$ smaller than experiment. To account for this, we shift our pressures such that $P=0$ corresponds to the experimental volume, and the lattice and internal parameters as a function of pressure are shown in Fig. 2 . As the pressure is increased, the parameters decrease in a smooth way, which contrasts with the experimental observation in Ref. [5, which seemed to indicate a sharp drop in the c parameter for $P>6.5 \mathrm{kbar}$ [37].

The fitted exchange parameters as functions of pressure, charge dosing, the VCA, and $U$ are reported in Table [. We find that $J_{1}$ becomes more ferromagnetic with increasing pressure, electron doping, and decreasing $U$, and that it is also the most sensitive to these parameters. At $28 \mathrm{kbar}$ of pressure, the absolute value of $J_{1}$ grows by $3.0 \mathrm{meV} \sim 35 \mathrm{~K}$ for $U=3.12 \mathrm{eV}$ and $4.5 \mathrm{meV} \sim 52 \mathrm{~K}$ for $U=4.7 \mathrm{eV}$. In Ref. 21, the critical transition temperature (Néel or Curie, depending on the magnetic state 

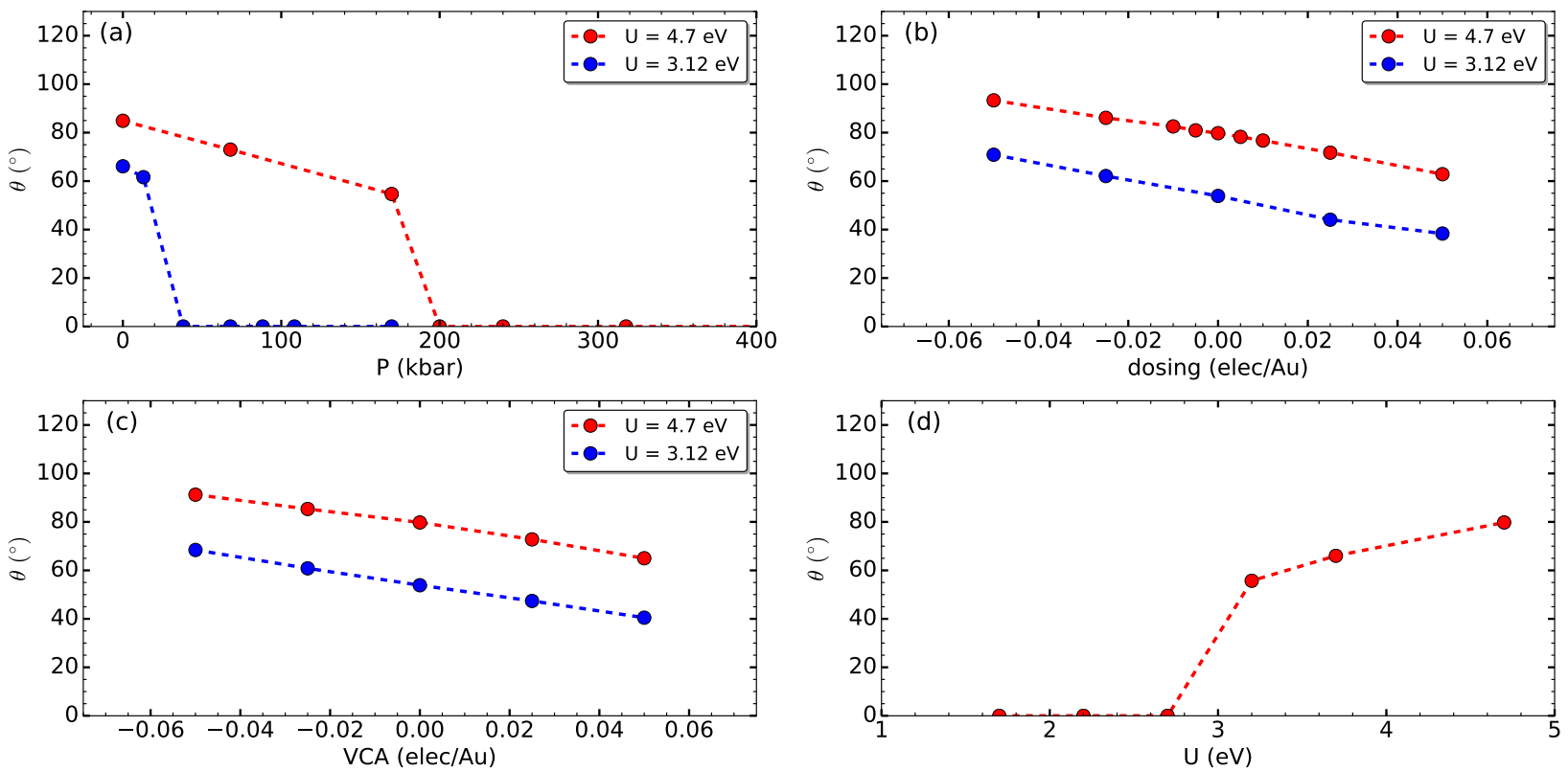

FIG. 4. (Color online) The spiral angle $\theta$ as a function of (a) pressure, (b) charge dosing, (c) the VCA, and (d) $U$. Panels (a), (b), and (c) include results for $U=3.12 \mathrm{eV}$ and $4.7 \mathrm{eV}$, see legend. The angle $\theta$ is obtained by minimizing the $J_{1}-J_{2}-J_{3}-J_{4}$ model.

at the given pressure) increases by $\sim 40 \mathrm{~K}$ going from zero pressure to $28 \mathrm{kbar}$, so these changes in $J_{1}$ are of the correct order.

To evaluate the spiral-to-ferromagnetic phase transition in the context of the phase diagrams in Fig. 1, we plot the scaled exchange parameters, $j_{i} \equiv J_{i} /\left|J_{1}\right|$, in Fig. 3. We find that in all cases $j_{2}$ is always antiferromagnetic and is the most sensitive to pressure, doping, and variation in $U . j_{2}$ decreases with increasing pressure, electron doping, and decreasing $U$. The exchange couplings $j_{3}$ and $j_{4}$ are closer to zero, and do not respond to pressure, doping, and $U$ in the same way as $j_{2}$. Applying pressure suppresses $j_{3}$, and for $U=4.7 \mathrm{eV}$ the coupling becomes slightly antiferromagnetic for $P \gtrsim 170 \mathrm{kbar}$. Charge dosing and VCA doping have a stronger effect, where $j_{3}$ becomes more ferromagnetic with hole doping (for $U=4.7 \mathrm{eV}$, the coupling becomes enhanced at the large hole doping of -0.05 electrons per $\mathrm{Au}$ ) and turns antiferromagnetic with electron doping. However, $j_{3}$ is insensitive to $U$, staying close to zero for all considered values. $j_{4}$, unlike $j_{3}$, remains ferromagnetic in all cases, and often $\left|j_{4}\right|>\left|j_{3}\right|$. Pressure affects $j_{4}$ in a similar way as $j_{3}$, and for $U=4.7 \mathrm{eV}$ so does doping, but for $U=3.12 \mathrm{eV}$, $j_{4}$ is less sensitive to doping than $j_{3}$. Finally, decreasing $U$ suppresses $j_{4}$, going from moderate ferromagnetic coupling at $U=4.7 \mathrm{eV}$ to being nearly zero at $U=1.2 \mathrm{eV}$. This indicates that measuring the fourth neighbor interplanar coupling in $\mathrm{MnAu}_{2}$ would yield information about the strength of the electronic correlations in this material.

Next we plot $\theta$ as a function of pressure, charge dosing,
VCA doping, and $U$ in Fig. 4. We obtain $\theta$ by minimizing Eq. (2) with the fitted exchange parameters [38]. We find that $\theta$ is quite sensitive to pressure, doping, and $U$. As expected, applying pressure decreases $\theta$ in agreement with Ref. 5, and at a critical pressure this induces a phase transition, which is in the range $12.977 \leq P \leq 38.109$ kbar for $U=3.12 \mathrm{eV}$ and $169.709 \leq P \leq 200 \mathrm{kbar}$ for $U=4.7 \mathrm{eV}$. This indicates that measurements of the critical pressure provides information about the strength of the electronic correlations. In addition, for both charge dosing and VCA doping the rate of change of $\theta$ is independent of $U$, and $\theta$ decreases with electron doping and increases with hole doping. Finally, in Fig. 4(d) we find that $\theta$ decreases with decreasing $U$, and eventually collapses to a ferromagnetic state.

The evolution of the magnetic coupling of $\mathrm{MnAu}_{2}$ when subjected to pressure, doping, and variation in $U$ can be further visualized using the contour plot in Fig. 1 (d). We take the exchange parameters $j_{3}$ and $j_{4}$ and plot them as parametric curves on top of the contour plot, which captures how they vary with pressure, doping, and $U$. For each point, we also compare the fitted $j_{2}$ with the critical $j_{2}^{c}$ that marks the spiral-toferromagnetic phase transition. If $j_{2}<j_{2}^{c}$, we plot the point as a circle, indicating that the system is ferromagnetic, and if $j_{2} \geq j_{2}^{c}$ we plot the point as a star, which represents spiral order. This procedure yields Fig. 5, and on each panel we give a reference point, which for panel (a) corresponds to the relaxed cell at experimental volume $(P=0)$, for panels $(b)$ and $(c)$ corresponds to the experimental lattice parameters with zero doping, and 

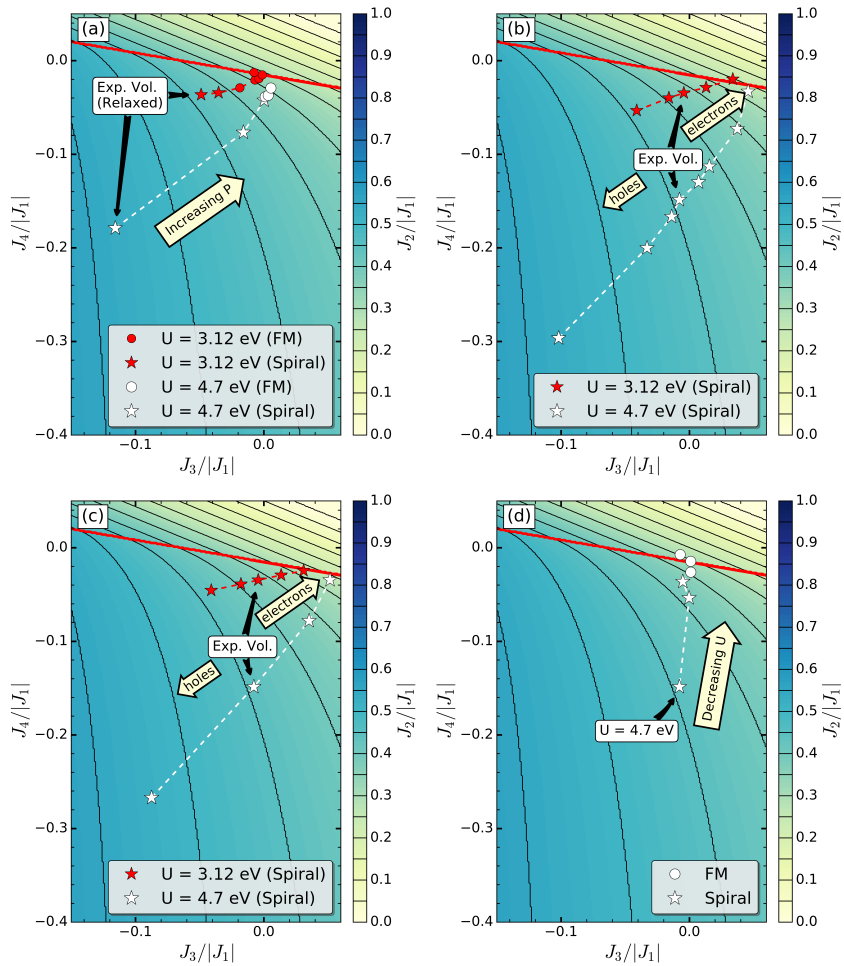

FIG. 5. (Color online) Parametric plots of the $\mathrm{MnAu}_{2}$ exchange parameters as a function of pressure, doping, and $U$. The parametric curves are visualized on top of the contour plot from Fig. 1(d). The plot marker shapes indicate the magnetic order of $\mathrm{MnAu}_{2}$, with stars corresponding to spiral order and circles to ferromagnetic order. See legend for the values of $U$. (a) Parametric curve as a function of pressure. The reference point is the $P=0$ unit cell, see Fig. 2 for lattice parameters. The arrow indicates the direction of increasing pressure. (b) Parametric curve as a function of charge dosing. The reference point is a unit cell with experimental lattice parameters and no doping. The arrows indicate the direction for increasing electron/hole doping. (c) Parametric curve as a function of the VCA. The reference point is a unit cell with experimental lattice parameters and no doping. The arrows indicate the direction for increasing electron/hole doping. (d) Parametric curve as a function of $U$. The reference point is a unit cell with experimental parameters and $U=4.7 \mathrm{eV}$. The arrow indicates the direction of decreasing $U$.

for panel (d) corresponds to experimental lattice parameters with $U=4.7 \mathrm{eV}$. The arrows with text indicate the direction that the parametric curves evolve, so following the curve from the reference point in panel (a) shows how the parameters change with increasing pressure, in panels (b) and (c) one direction is electron doping and the other is hole doping, and for panel (d) $U$ decreases along the curve. If, while following the curve, the marker symbol changes from a star to a circle, this corresponds to a phase transition. If this change in symbols happens while south of the red line, then the phase transition is first order, and if it happens north of the red line, then it is a second order.
The parametric path for $\mathrm{MnAu}_{2}$ under pressure in Fig. 5(a) shows that the spiral-to-ferromagnetic transition is first order for both values of $U$. So, for ideal, stoichiometric $\mathrm{MnAu}_{2}$ samples, we expect the pressureinduced phase transition to be of first order, in agreement with Ref. 22. Decreasing $U$ at ambient pressure also induces a first order phase transition. In contrast, for our considered doping levels $\mathrm{MnAu}_{2}$ does not collapse to the ferromagnetic state. Doping with holes stabilizes the spiral state and drives the system deeper into the first-order parameter region, while doping with electrons moves $\mathrm{MnAu}_{2}$ closer to ferromagnetism and also towards a second-order phase transition. Also, the stronger the correlations, the more dramatic the shifts in parameter space with doping. For both $U=3.12 \mathrm{eV}$ and $4.7 \mathrm{eV}$ an electron doping of 0.05 electrons per Au places the system at the dividing line between first and second order, and it would be expected that the application of pressure for this system would induce a second order transition.

We check the prediction of inducing a second order phase transition by calculating the pressure dependence of $\mathrm{MnAu}_{2}$ with $U=3.12 \mathrm{eV}$ and a charge dosing level of 0.07 electrons per Au. The exchange parameters obtained from these calculations are reported in Table II and the scaled exchange parameters, pressure dependence of $\theta$, and a parametric plot of the exchange parameters are plotted in Fig. 6. As shown in Fig. 6(a) the initial charge dosing biases $j_{2}$ to be less antiferromagnetic and for $j_{3}$ to become more antiferromagnetic. For the range of pressures considered, $j_{2}$ decreases with increasing pressure while $j_{3}$ and $j_{4}$ are unaffected. Fig. 6(b) shows the effect this has on the $\theta$ vs pressure curve, where the trend of the charge-dosed curve is consistent with a second order phase transition, particularly when compared with the first order transition of the undoped curve included for comparison. The parametric curve in Fig. 6.(c) shows that the charge-dosed pressure-dependent path in exchange parameter space is north of the dividing line, confirming the prediction that applying pressure to electron doped $\mathrm{MnAu}_{2}$ can induce a second-order phase transition.

We can use the result that applying pressure to electron-doped $\mathrm{MnAu}_{2}$ induces a second order phase

\begin{tabular}{ccccc}
\hline $\begin{array}{c}\text { Pressure } \\
(\mathrm{kbar})\end{array}$ & $J_{1}$ & \multicolumn{4}{c}{$J_{2}$} & $J_{3}$ & $J_{4}$ \\
\hline 0.000 & -33.5978 & 10.2441 & 0.8442 & -0.6193 \\
2.000 & -33.9957 & 10.2223 & 0.8197 & -0.6252 \\
6.000 & -34.8238 & 10.1037 & 0.8679 & -0.6570 \\
10.000 & -35.6126 & 10.0322 & 0.8756 & -0.6331 \\
18.000 & -37.0886 & 9.9025 & 0.9327 & -0.6302 \\
20.000 & -37.4883 & 9.8482 & 0.9131 & -0.6498 \\
24.000 & -38.1828 & 9.7781 & 0.9236 & -0.6241 \\
\hline
\end{tabular}

TABLE II. Values of the fitted exchange parameters for the simultaneous pressure and charge dosing calculations. Results are for a charge dosing value of 0.07 electrons per $\mathrm{Au}$ and $U=3.12 \mathrm{eV}$. 

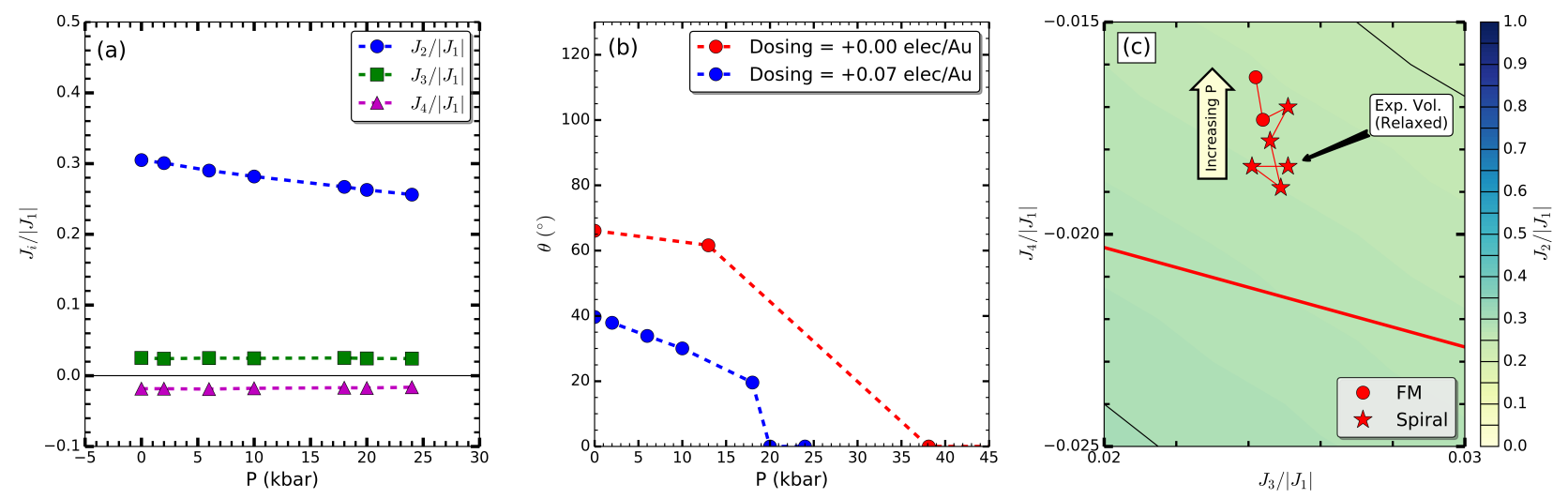

FIG. 6. (Color online) Plots showing the results of calculations with simultaneous charge dosing and applied pressure. The calculations were performed using $U=3.12 \mathrm{eV}$ and a charge dosing of 0.07 electrons per Au. (a) The exchange parameters $J_{2} /\left|J_{1}\right|, J_{3} /\left|J_{1}\right|$, and $J_{4} /\left|J_{1}\right|$, see legend, as a function of pressure. (b) The spiral angle $\theta$ as a function of pressure with and without charge dosing, see the legend. (c) Parametric plot of the exchange parameters as a function of pressure visualized on top of the contour plot from Fig. 1(d). The plot markers indicate the type of magnetic order, see the legend. The reference point is the relaxed $P=0$ unit cell. The arrow indicates the direction of increasing pressure.

transition to resolve the contradiction in the pressure experiments. In Ref. 21, where the authors found a second order phase transition for $\mathrm{MnAu}_{2}$, they reported that there were trace amounts of $\mathrm{MnAu}$ in their sample, such that the system was $34.3 \% \mathrm{Mn}$ and $65.7 \% \mathrm{Au}$. This excess of Mn in their sample can lead to an effective electron doping. Let us assume that the excess $\mathrm{Mn}$ atoms are also in the $2+$ ionic charge state, and that the extra electrons they donate are spread homogeneously over the system. This would mean that each unit cell of $\mathrm{MnAu}_{2}$ would have 2 electrons per $\mathrm{Mn} \cdot(34.3 / 33.3)=$ 2.06 electrons per $\mathrm{Mn}$, or an excess of 0.03 electrons per $\mathrm{Au}$. Assuming that $U=3.12 \mathrm{eV}$ [39] and comparing with the electron dosing curve in Fig. 5(b), an extra 0.03 electrons per $\mathrm{Au}$ would shift the sample near the second order transition region of the parameter space, which as we've seen can lead to a second order phase transition upon application of pressure. While the $\mathrm{MnAu}$ domains are not likely to uniformly dope the material, we see how the variations in sample quality can influence the kind of phase transition. This both stresses the importance of controlling for impurities in $\mathrm{MnAu}_{2}$, and that the magnetic properties are also amenable to tuning.

\section{CONCLUSIONS}

We studied how the magnetic exchange in $\mathrm{MnAu}_{2}$ changes upon application of pressure and doping using a series of DFT calculations, as well as varying the electronic correlations with a Hubbard-like $U$. We found that the proper model for these interactions is the onedimensional Heisenberg model with coupling between the first four Mn planes (the $J_{1}-J_{2}-J_{3}-J_{4}$ model), which contrasts with previous studies that only considered interactions between the first two planes. Within this model, transitions between the ferromagnetic and spiral phases can be either first or second order, and $J_{3}$ and $J_{4}$ control the order of the phase transition. Our analysis finds that ideal, stoichiometric $\mathrm{MnAu}_{2}$ sits near a dividing line in $\left(J_{3}, J_{4}\right)$ space that separates first- and second-order phase transitions. Applying pressure collapses the spiral state as expected, and for ideal, stoichiometric $\mathrm{MnAu}_{2}$ the transition is first order. Hole doping the material further stabilizes the spiral and the tendency towards a first-order transition. In contrast, electron doping the material makes the spiral less stable and moves the system in $\left(J_{3}, J_{4}\right)$ space closer to a secondorder transition. We confirm with additional DFT calculations that applying pressure to electron-doped $\mathrm{MnAu}_{2}$ will induce a second-order phase transition. Therefore, impurities that provide an effective electron doping, such as excess $\mathrm{Mn}$, can cause the spiral-to-ferromagnetic phase transition to become second order under pressure. These results indicate that $\mathrm{MnAu}_{2}$ is tunable, where the kind of phase transition can be set and the spiral angle controlled through both pressure and doping, which can include gate voltages. The ability to control the spiral angle holds promise for integration of $\mathrm{MnAu}_{2}$ in spintronics devices, such as in a spin valve. Attaching a thin film of $\mathrm{MnAu}_{2}$ to the free magnetic layer of a spin valve and then applying a gate voltage could allow for a current-free manipulation of the relative orientation between the free and pinned magnetic layers, which would reduce Joule heating and could help in developing smaller-scale electronics devices.

\section{ACKNOWLEDGMENTS}

J.K.G. thanks Igor Mazin and Konrad Bussmann for useful discussions regarding this project, and acknowl- 
edges the support of the NRC program at NRL.

\section{SUPPLEMENTARY MATERIALS}

The supplemental materials for this report are provided in the IPython notebook format. The notebook file and the data used for analysis are provided at https://github.com/jkglasbrenner/ipython_ nb_collapse_and_control_of_the_mnau2_spin_ spiral_state_through_pressure_and_doping. For a readable HTML version of the notebook, download the following file and open it in your web-browser: https://github.com/jkglasbrenner/ipython_ nb_collapse_and_control_of_the_mnau2_spin_ spiral_state_through_pressure_and_doping/raw/ master/ipython_nb_MnAu2_pressure_supplemental_ material.html
[1] A. J. Meyer and P. Taglang, J. Phys. Radium 17, 457 (1956).

[2] A. Herpin, P. Meriel, and J. Villain, Comptes Rendus 249, 1334 (1959).

[3] A. Herpin and P. Meriel, J. Phys. Radium 22, 337 (1961).

[4] H. Samata, N. Sekiguchi, A. Sawabe, Y. Nagata, T. Uchida, and M. D. Lan, J. Phys. Chem. Sol. 59, 377 (1998).

[5] F. A. Smith, C. C. Bradley, and G. E. Bacon, J. Phys. Chem. Sold. 27, 925 (1966).

[6] A. Handstein, U. K. Rößler, B. Idzikowski, N. Kozlova, K. Nenkov, K. H. Müller, A. Kreyssig, M. Loewenhaupt, A. Heinemann, A. Hoell, and N. Stüßer, J. Mag. Mag. Mater. 290-291, Part 2, 1093 (2005).

[7] W. E. Evenson and S. H. Liu, Phys. Rev. Lett. 21, 432 (1968).

[8] M. A. Ruderman and C. Kittel, Phys. Rev. 96, 99 (1954).

[9] T. Kasuya, Prog. Theor. Phys. 16, 45 (1956).

[10] K. Yosida, Phys. Rev. 106, 893 (1957).

[11] I. Dzyaloshinsky, J. Phys. Chem. Sol. 4, 241 (1958).

[12] T. Moriya, Phys. Rev. 120, 91 (1960).

[13] P. Bak and M. H. Jensen, J. Phys. C 13, L881 (1980).

[14] O. Nakanishi, A. Yanase, A. Hasegawa, and M. Kataoka, Sol. St. Comm. 35, 995 (1980).

[15] M. Kataoka and O. Nakanishi, J. Phys. Soc. Jpn. 50, 3888 (1981).

[16] U. Enz, J. Appl. Phys. 32, S22 (1961).

[17] T. Nagamiya, J. Appl. Phys. 33, 1029 (1962).

[18] L. Udvardi, S. Khmelevskyi, L. Szunyogh, P. Mohn, and P. Weinberger, Phys. Rev. B 73, 104446 (2006).

[19] J. K. Glasbrenner, K. M. Bussmann, and I. I. Mazin, Phys. Rev. B 90, 144421 (2014).

[20] N. P. Grazhdankina and K. P. Rodionov, Sov. Phys. JETP 16, 1429 (1963).

[21] R. C. Wayne and F. A. Smith, J. Phys. Chem. Sol. 30, 183 (1969).

[22] R. Adiatullin and I. G. Fakidov, Sov. Phys. Sol. St. 12, 2547 (1971).

[23] A. Pimpinelli, E. Rastelli, and A. Tassi, J. Phys.: Cond. Mat. 1, 7941 (1989).

[24] R. Zinke, J. Richter, and S.-L. Drechsler, J. Phys.: Cond. Mat. 22, 446002 (2010).

[25] ELK FP-LAPW Code http://elk.sourceforge.net/].

[26] G. Kresse and J. Hafner, Phys. Rev. B 47, 558(R) (1993).

[27] G. Kresse and J. Furthmüller, Phys. Rev. B 54, 11169 (1996).

[28] J. P. Perdew and Y. Wang, Phys. Rev. B 45, 13244 (1992).
[29] A. I. Liechtenstein, V. I. Anisimov, and J. Zaanen, Phys. Rev. B 52, R5467(R) (1995).

[30] K. Adachi, K. Sato, H. Watarai, and T. Ido, J. Phys. Soc. Jpn. 32, 572 (1972).

[31] L. W. Hart and J. L. Stanford, J. Appl. Phys. 41, 2523 (1970).

[32] The experimental pressure studies of $\mathrm{MnAu}_{2}$ do not find changes in the crystal structure prior to the spiralto-ferromagnetic phase transition. For this reason, we only investigate tetragonal unit cells and do not consider whether other structures become competitive at higher pressures, since they will not impact the phase transition.

[33] See Supplemental Material at [URL will be inserted by publisher] for our raw data and a Jupyter (formerly IPython) notebook that describes the published research in full detail. The goal of including this material is so that readers can verify and reproduce all the presented results. The notebook contains all the Python code used to analyze our DFT calculations and produce the figures and tables contained in the manuscript. The notebook also contains some additional calculations and diagrams not presented in the main text that, while not essential for our arguments, may be of interest to the reader. Specifically, the notebook contains: (1) an annotated analysis of the results of structural optimizations performed in VASP, (2) a full and annotated derivation of the analytic results of the one-dimensional Heisenberg model, (3) details of how we fit our results to the one-dimensional $J_{1}-J_{2}-J_{3}-J_{4}$ Heisenberg model, and (4) how we created the contour plots, phase diagrams, and visualized the exchange parameters and spiral angle $\theta$ as a function of pressure, doping, and $U$.

[34] Convergence of the magnetocrystalline anisotropy energy in the $\mathrm{MnAu}_{2}$ unit cell was achieved with the following parameters in ELK: $\mathrm{k}$-mesh $=28 \times 28 \times 28$, nempty $=$ 12 , rgkmax $=8.0$, gmaxvr $=16$, lmaxapw $=14$, lmaxvr $=14$, and the smearing function width was set to 0.0001 Ha.

[35] Y. Nagata, T. Hagii, H. Samata, T. Uchida, S. Abe, C. Fan Sung, and M. Der Lan, J. Al. Comp. 284, 47 (1999).

[36] A. Handstein, K. Nenkov, U. K. Rössler, and K.-H. Müller, J. Appl. Phys. 87, 5789 (2000).

[37] There is a large error in measuring $c$ as a function of pressure in Ref. 15, so it is likely that the reported drop is not a real effect.

[38] We note that an alternative approach is to interpolate $E(\theta)$ and find the minimum, and away from phase tran- 
sitions these two methods are in good agreement (see Supplemental Material [33]). However, near a phase transition the energy wells become shallow and the interpolations are no longer reliable, so for consistency we report results obtained by minimizing Eq. (2).
[39] This choice of $U$ yields a $\theta<60^{\circ}$ and a critical transition pressure $P \sim 20-40 \mathrm{kbar}$, in reasonable agreement with experiment. 